\title{
Erratum to: Impacts of rising air temperatures and emissions mitigation on electricity demand and supply in the United States: a multi-model comparison
}

\author{
James McFarland ${ }^{4} \cdot$ Yuyu Zhou $^{1} \cdot$ Leon Clarke $^{1} \cdot$ Patrick Sullivan $^{3}$ • \\ Jesse Colman ${ }^{3}$ - Wendy S. Jaglom ${ }^{2}$ - Michelle Colley ${ }^{2} \cdot$ Pralit Patel $^{1}$ • \\ Jiyon Eom ${ }^{1}$ • Son H. Kim ${ }^{1}$ - G. Page Kyle ${ }^{1}$ Peter Schultz ${ }^{2}$ • \\ Boddu Venkatesh $^{2}$ • Juanita Haydel ${ }^{2}$. Charlotte Mack ${ }^{2}$. Jared Creason ${ }^{4}$
}

Published online: 7 July 2015

(C) Springer Science+Business Media Dordrecht 2015

Erratum to: Climatic Change (2015) 131:111-125

DOI 10.1007/s10584-015-1380-8

In the initial online publication figures 1 and 4 both appeared twice. The article has been updated to correct this mistake

The online version of the original article can be found at http://dx.doi.org/10.1007/s10584-015-1380-8.

\section{James McFarland}

mcfarland.james@epa.gov

1 Joint Global Change Research Institute, PNNL, College Park, MD, USA

2 ICF International, Fairfax, VA, USA

3 National Renewable Energy Laboratory, Golden, CO, USA

4 U.S. Environmental Protection Agency, Washington, DC, USA 